Of all patients with clinically active disease at baseline $(\mathrm{n}=20), 5$ achieved clinical response $(25 \%)$, an additional 4 achieved clinical remission (20\%).
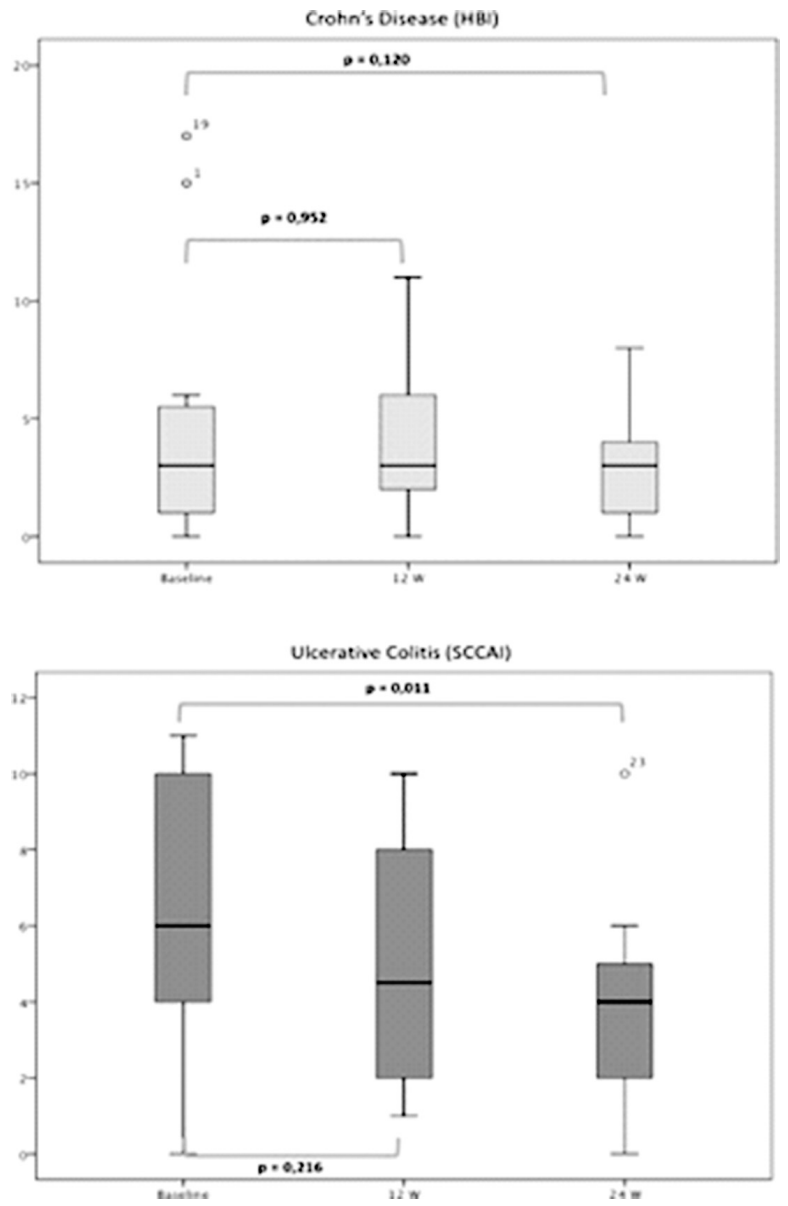

Abstract PWE-005 Figure 1

Conclusions In a real life setting, increasing dosing frequency in patients with sub-optimal response to VDZ is effective in approximately half of patients and should be considered as an intervention.

\section{PWE-006 SMOKING IN UC IS ASSOCIATED WITH DECREASED THIOPURINE USE BUT NOT STEROID DEPENDENCY OR COLECTOMY}

Jonathan *. St George's NHS Foundation Trust, South Croydon, UK

\subsection{6/gutjnl-2018-BSGAbstracts. 138}

Introduction Whilst smoking is established as a protective modifiable environmental risk factor for the development of Ulcerative Colitis (UC), the evidence for its impact on subsequent disease activity is conflicting. We therefore aimed to investigate the impact of smoking on clinical outcomes in the disease course of UC.

Methods Using a nationally representative clinical practice research database (CPRD), we identified incident cases of UC diagnosed between 2005 and 2014. Patients were grouped as: smokers, never-smokers or ex-smokers at UC diagnosis based on medical record codes for smoking status in the two years preceding UC diagnosis. Medical record codes were also examined to determine change in smoking status following diagnosis. We compared corticosteroid dependency (as defined in ECCO guidelines), thiopurine use and colectomy rates between these defined groups. Survival analysis, Cox proportional hazards analysis and logistic regression were used determine the risk of first thiopurine use, corticosteroid dependency and colectomy given smoking status.

Abstract PWE-006 Table 1 Univariate and multivariate Cox regression analysis for risk of Thiopurine use in patients with Ulcerative Colitis

\begin{tabular}{|c|c|c|c|c|c|c|}
\hline & \multicolumn{3}{|c|}{$\begin{array}{c}\text { Univariate analysis } \\
n=4058\end{array}$} & \multicolumn{3}{|c|}{$\begin{array}{c}\text { Multivariate analysis } \\
n=1,587\end{array}$} \\
\hline & HR & $95 \% \mathrm{CI}$ & pvalue & HR & $95 \% \mathrm{CI}$ & p value \\
\hline \multicolumn{7}{|l|}{ Smoking status" } \\
\hline Never Smoker & 1 & - & - & - & - & - \\
\hline Persistent Smoker & 0.61 & $0.44-0.86$ & 0.005 & 0.52 & $0.27-0.97$ & 0.041 \\
\hline Persistent Ex-Smaker & 0.84 & $0.71 \cdot 0.99$ & 0.046 & 1.00 & $0.75-1.34$ & 0.95 \\
\hline New Smoker Post UC & 0.86 & $0.58-1.28$ & 0.485 & 0.84 & $0.43-1.62$ & 0.60 \\
\hline New Ex-Smoker Post UC & 0.96 & $0.69-1.33$ & 0.819 & 1.07 & $0.62-1.85$ & 0.80 \\
\hline Relapsed Smoker Post UC. & 1.23 & $0.90-1.68$ & 0.181 & 1.01 & $0.59-1.73$ & 0.94 \\
\hline \multicolumn{7}{|l|}{ Sex } \\
\hline Female & 1 & - & - & - & - & - \\
\hline Male & 1.26 & $1.08-1.45$ & 0.002 & 1.30 & $1.01-1.68$ & 0.040 \\
\hline \multicolumn{7}{|l|}{ Age at 180 diagnosis ${ }^{b}$} \\
\hline A1 & 1 & - & - & - & - & - \\
\hline A2 & 0.53 & $0.31-0.89$ & 0.017 & 2.39 & $0.72-7.89$ & 0.15 \\
\hline $\mathrm{A}_{3}$ & 0.26 & $0.15-0.44$ & 0.000 & 1.11 & $0.33-3.65$ & 0.86 \\
\hline \multicolumn{7}{|l|}{ Era of liBO diagnosis } \\
\hline $2005 / 2006$ & 1 & - & - & - & - & - \\
\hline $2007 / 2008$ & 1.09 & $0.87-1.36$ & 0.429 & 0.92 & $0.63-1.35$ & 0.69 \\
\hline $2009 / 2010$ & 1.06 & $0.84-1.33$ & 0.593 & 1.10 & $0.76-1.59$ & 0.68 \\
\hline $2011 / 2012$ & 1.45 & $1.15-1.82$ & 0.001 & 1.49 & $1.02-2.18$ & 0.035 \\
\hline $2013 / 2014$ & 1.50 & $1.15-1.95$ & 0.003 & 1.51 & $0.90-2.55$ & 0.11 \\
\hline \multicolumn{7}{|l|}{ BMI category' } \\
\hline Underweight & 0.96 & $0.57-1.63$ & 0.898 & 0.58 & $0.30-1.11$ & 0.10 \\
\hline Normal & 1 & - & - & - & - & - \\
\hline Overweight & 0.77 & $0.62-0.95$ & 0.016 & 0.89 & $0.67-1.19$ & 0.44 \\
\hline Obese & 0.72 & $0.56-0.92$ & 0.009 & 0.92 & $0.65-1.31$ & 0.66 \\
\hline \multicolumn{7}{|l|}{ Social deprivation" } \\
\hline IMO 1 & 1 & - & - & & & - \\
\hline IMO 2 & 0.86 & $0.65-1.13$ & 0.306 & 0.87 & $0.61-1.24$ & 0.45 \\
\hline IMO 3 & 0.97 & $0.72-1.29$ & 0.838 & 0.95 & $0.64-1.40$ & 0.80 \\
\hline IMO 4 & 0.88 & $0.66-1.18$ & 0.412 & 1.01 & $0.70-1.46$ & 0.95 \\
\hline IMOS & 0.98 & $0.71-1.36$ & 0.940 & 0.83 & $0.56-1.24$ & 0.38 \\
\hline \multicolumn{7}{|l|}{ Co-morbidities' } \\
\hline IBS & 0.79 & $0.63-1.00$ & 0.055 & 0.60 & $0.40-0.88$ & 0.011 \\
\hline Depressian & 0.75 & $0.57-0.99$ & 0.045 & 0.76 & $0.49-1.20$ & 0.24 \\
\hline \multicolumn{7}{|l|}{ IBD medications } \\
\hline Oral 5-ASA & 2.50 & $2.06-3.03$ & $<0.001$ & 1.57 & $1.16-2.13$ & 0.004 \\
\hline Corticosteroids & 8.57 & $6.86-10.71$ & $<0.001$ & $x$ & $\mathrm{x}$ & $\mathrm{x}$ \\
\hline \multicolumn{7}{|l|}{ Severity Indices" } \\
\hline Early Corticosteroid Use & 3.68 & $3.19-4.26$ & 0.008 & 1.43 & $1.09-1.87$ & 0.003 \\
\hline Colectomy & 3.20 & 2.49 .4 .12 & 0.007 & 1.76 & $1.16-2.67$ & 0.007 \\
\hline Corticosteroid Dependence & 5.62 & $4.85-6.51$ & $<0.001$ & 2.02 & $1.55-2.64$ & $<0.001$ \\
\hline $\begin{array}{l}\text { Corticosteroid Flares Per Year } \\
0\end{array}$ & 1 & - & - & - & - & - \\
\hline $0-1$ & 8.39 & $6.69-10.51$ & $<0.001$ & 5.11 & $3.34-7.81$ & $<0.001$ \\
\hline$>1$ & 13.05 & $9.40-18.10$ & $<0.001$ & 6.15 & $3.22-11.72$ & $<0.001$ \\
\hline
\end{tabular}

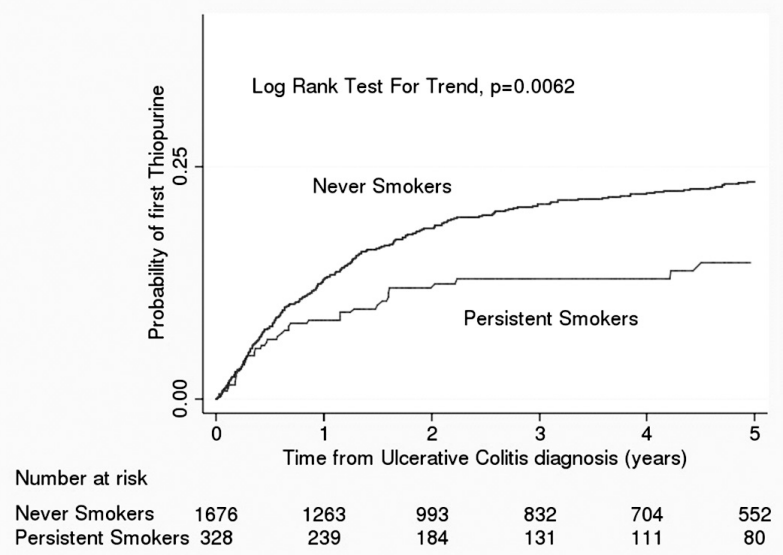

Abstract PWE-006 Figure 1 Kaplan Meier Curve: Progression to Thiopurine Use in Smokers and Never Smokers in Ulcerative Colitis 
Result We identified 4069 cases of UC over the study period. There were 1678 never smokers (41\%), 329 smokers (8\%), 1541 ex-smokers (38\%) and 521 patients whose follow-up smoking status changed (13\%). Multivariate regression analysis, adjusting for all covariates listed in table 1, demonstrated smokers had a significantly lower risk of thiopurine use compared to both never smokers (HR 0.52, 95\% CI 0.27-0.97, $\mathrm{p}=0.04$ ) and ex-smokers (HR 0.51, 95\% CI 0.27-0.98, $\mathrm{p}=0.04)$. In contrast there was no difference in corticosteroid dependency (OR 0.83 , 95\% CI $0.44-1.75$ ) or rates of colectomy (HR 0.4 , 95\% CI 0.53-3.02) in a multivariate analysis. Conclusions Smoking is associated with a decreased requirement for thiopurines however it does not impact on the risk of corticosteroid dependency or colectomy. The risks associated with smoking outweigh any benefits and smoking cessation should be encouraged.

\section{PWE-007 A SYSTEMATIC REVIEW OF OUTCOMES AND ADVERSE EVENTS FOR RANDOMISED CONTROLLED TRIALS IN CROHN'S DISEASE}

${ }^{1}$ Ms Heather Catt*, ${ }^{2}$ Dyfrig Hughes, ${ }^{1,3}$ Keith Bodger, ${ }^{1}$ Jamie Kirkham. ${ }^{1}$ University Of Liverpool (MRC Trials Hub), Liverpool, UK; ${ }^{2}$ University of Bangor, Bangor, UK; ${ }^{3}$ Aintree University Hospital NHS Trust, Liverpool, UK

\subsection{6/gutjnl-2018-BSGAbstracts.139}

Introduction Despite major progress in drug development for Crohn's disease (CD) and advances in trial methodology, there is no internationally recognised core outcome set (COS). Poor standardisation in outcome reporting may impact negatively on translation of trials into practice. The suitability of traditional disease activity indices as primary end-points has been challenged, with growing interest in objective measures of inflammation. We undertook a systematic review to explore heterogeneity and time trends in the reporting of efficacy and safety outcomes in placebo-controlled randomised controlled trials (RCTs) of patients with CD.

Methods We searched MEDLINE, EMBASE, CINAHL and Cochrane Library from their inception to November 2015, for RCTs of adult CD patients with treated with medical or surgical therapies. We extracted information on efficacy and safety outcomes, definitions of end-points, and measurement instruments. To explore temporal trends studies were stratified by publication date (pre-2009 and 2009-onwards).

Results 181 RCTs comprising 23850 patients. Trial focus: Induction of remission, 110 trials (60.8\%), 104 medical and 6 surgical interventions. Maintenance of remission, 71 trials (39.2\%). Biologics were intervention of interest in $33.7 \%$, as either monotherapy or part of a combination therapy. 92.3\% of trials reported clinical efficacy outcomes as a primary or secondary endpoint. CDAI was the dominant index, used to determine clinical response or remission in $63.5 \%$ of trials. However, there was heterogeneity, with 35 definitions of response or remission. CDAI $<150$ was the commonest endpoint, but reporting reduced between periods $(46.4 \%$ to $41.1 \%$ of trials), whilst CDAI100 reporting increased $(16.8 \%$ to $30.4 \%)$. Reporting of objective measures of inflammation increased over time, but with lack of standardisation. Reporting of both histologic and endoscopic outcomes increased, from $3.2 \%$ to $12.5 \%$ and from $14.4 \%$ to $30.4 \%$ of RCTs, respectively. Biomarker reporting increased from $33.3 \%$ to
$40.6 \%$ of trials. Patient-reported outcome measures (PROMs) were reported in $41.4 \%$ of trials with growth in reporting from $39.2 \%$ to $46.4 \%$. Safety outcomes were reported explicitly in $35.4 \%$ of trials and reporting increased from $32.8 \%$ to $41.1 \%$.

Conclusions As expected, the CDAI was the dominant composite index reported but there was significant variation in the selection and definition of clinical trial end-points in RCTs for CD between studies, and over time. Despite growth in reporting of objective measures of inflammation and in PROMs, there is much heterogeneity and lack of standardisation. This highlights the need for international researchers and clinicians to develop a COS for comparative effectiveness research in CD.

\section{PWE-008 CLINICAL OUTCOMES OF USTEKINUMAB IN RESISTANT CROHN'S DISEASE: UK IBD TERTIARY REFERRAL CENTRE 'REAL-WORLD' EXPERIENCE}

Simon Peter Borg-Bartolo*, Karen Kemp, Robert P Willert, Alistair J Makin, Scott Edward Levison. Department of Gastroenterology, Manchester Royal Infirmary, Manchester University NHS Foundation Trust, Manchester, UK

\subsection{6/gutjnl-2018-BSGAbstracts. 140}

Introduction Ustekinumab (UST) binds to the p40 subunit of IL12 and IL23 to prevent IL12RB1 cell-surface receptor activation and thus inhibits downstream inflammatory signalling. It is approved for moderately to severely active Crohn's disease (NICE TA456). We assessed the clinical outcomes and safety of UST in a 'real-world' cohort of refractory Crohn's disease patients treated at a single UK centre.

Methods We retrospectively collected data from the electronic records of Crohn's disease patients treated with UST at a single UK IBD tertiary referral centre. Patient demographics and adverse events were recorded. Clinical response to UST was evaluated at baseline and follow up using Harvey-Bradshaw Index (HBI) scores, $\mathrm{C}$ reactive protein (CRP), and faecal calprotectin (FC). Paired Student's T Tests were used to determine statistical significance.

Results 26 patients (mean age 36 years; age 18-62 years; M: $\mathrm{F}$ ratio $=1: 1.6)$ with a variety of Crohn's disease phenotypes $(\mathrm{L} 1=8 ; \mathrm{L} 2=6 ; \mathrm{L} 3=12)$ were treated with UST. 9 patients (35\%) had stricturing disease and 5 patients (19\%) penetrating disease. All patients had failed at least one anti-TNF agent. 15 patients $(58 \%)$ had failed two anti-TNF agents, and $11(42 \%)$ had failed an anti-TNF and subsequent vedolizumab therapy. 7 patients $(27 \%)$ received immunomodulatory co-therapy (AZA $=5 ; \mathrm{MTX}=2)$, and $11(42 \%)$ received bridging steroids.

12 week data was available for 20 patients. At 12 weeks, mean HBI significantly improved (5 vs 9; $\mathrm{p}<0.05$ ). There was reduction in mean FC (763 vs 1026 ; ns), but no change in mean CRP (14 vs 11 ; ns). 10 patients (50\%) demonstrated subjective and objective (FC +/-CRP +/- endoscopic) response to therapy. 6 of these patients received bridging steroids, of which all had reduced and 4 had completed their steroid course. Of all treated patients 2 discontinued UST (recurrence of a transitional cell carcinoma; primary nonresponse to therapy requiring surgery), and side effects were reported in 2 patients (Bell's Palsy; lower respiratory tract infection). 